Supporting Information

\title{
Photochemical Phase Transition versus Photochemical Phase Separation
}

\section{Synthesis and characterization of the liquid crystalline azobenzene dopant}

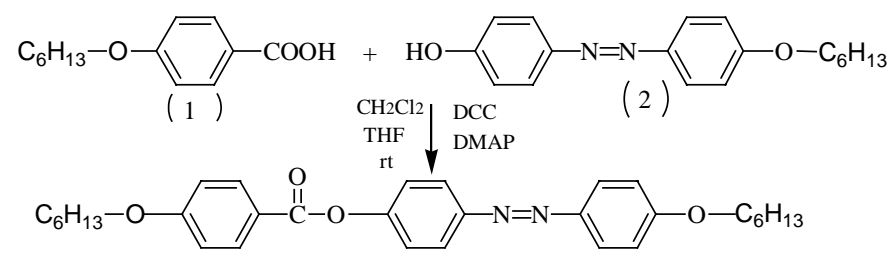

Literature methods were used to prepare compounds $\mathbf{1}$ and $\mathbf{2}$ in the above synthetic scheme. ${ }^{1,2}$ Details on the synthesis of the LC azo dopant are as follows. A solution of $\mathbf{1}(1.2 \mathrm{~g}, 5.4 \mathrm{mmol}), \mathbf{2}(1.6 \mathrm{~g}, 5.4$ $\mathrm{mmol}$ ) and 4-(dimethylamino)-pyridine (DMAP) $(0.07 \mathrm{~g}, 0.6 \mathrm{mmol})$ was prepared in a mixture of dry tetrahydrofuran (THF) $(20 \mathrm{~mL})$ and methylene chloride $\left(\mathrm{CH}_{2} \mathrm{Cl}_{2}\right) \quad(8.0 \mathrm{~mL})$. N, $\mathrm{N}^{\prime}-$ dicyclohexycarbodiimide (DCC) $(1.23 \mathrm{~g}, 6.0 \mathrm{mmol})$ was added in the solution after it was stirred at 0 ${ }^{\circ} \mathrm{C}$ for $1 \mathrm{~h}$. The resulting mixture was stirred for another $2 \mathrm{~h}$ at $0{ }^{\circ} \mathrm{C}$, then for $24 \mathrm{~h}$ at room temperature. The precipitate was filtered off, and the filtrate was dried under reduced pressure. The product was purified by column chromatography on silica gel (eluent: ethyl acetate/petroleum ether=1:5). Yield, 1.6 g (3.2 mmol, 59.3\%). MS (m/e): $502\left(\mathrm{M}^{+}\right) .{ }^{1} \mathrm{H}$ NMR $\left(\delta, \mathrm{CDCl}_{3}\right): 8.20-8.10(2 \mathrm{H}, \mathrm{d}), 8.00-7.93(4 \mathrm{H}, \mathrm{dd})$, 7.49-7.31 (2H, d), 7.05-6.92 (4H, dd), 4.10-3.98 (4H, m), 1.90-1.73 (4H, m), 1.55-1.22 (12H, m), 1.00$0.85(6 \mathrm{H} \mathrm{t})$.

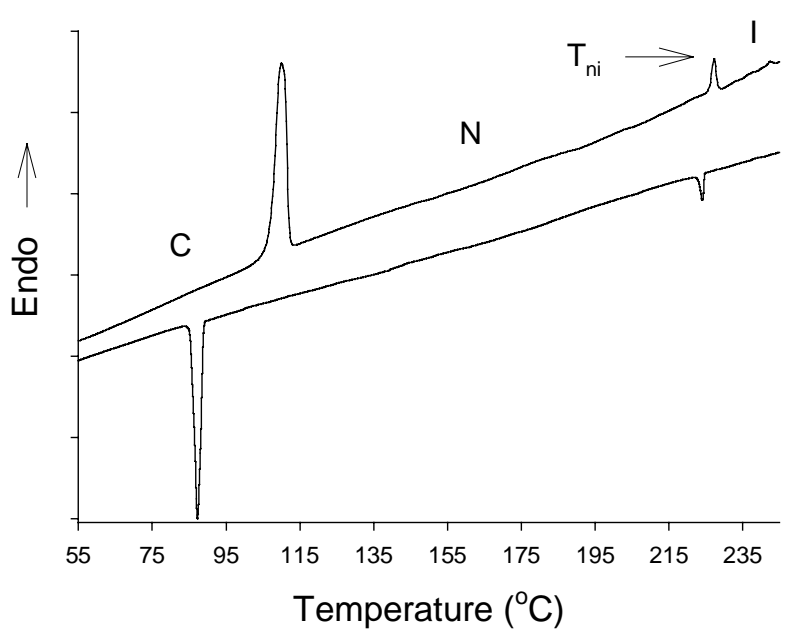

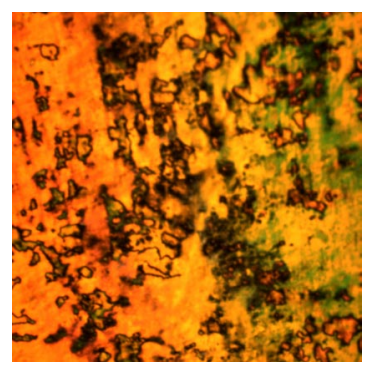

Figure S1. DSC heating and cooling curves of the LC azo dopant. The polarizing photomicrograph taken at $195{ }^{\circ} \mathrm{C}(64 \times)$ shows the nematic phase texture.

The azo dopant has been characterized using a number of techniques including differential scanning calorimetry (Perkin-Elmer DSC-7), polarizing optical microscopy (Leitz DMR-P), ${ }^{1} \mathrm{H}$ nuclear magnetic resonance (Bruker, AC-300), mass spectroscopy (Micromass ZAB-1F), infrared (Bomem MB-200 FTIR), UV-Vis spectroscopy (HP-8452A) and a X-ray diffractometer with a two-dimensional positionsensitive wire-grid detector (Bruker AXS). On the basis of the results, the azo dopant was found to have a nematic phase between 110 and $227^{\circ} \mathrm{C}$. Figure S1 shows the DSC heating and cooling curve 
$\left(2^{\text {nd }} \mathrm{scan}, 10{ }^{\circ} \mathrm{C} / \mathrm{min}\right)$ of the azo compound, together with a polarizing photomicrograph recorded at 195 ${ }^{\circ} \mathrm{C}$ that displays the texture of a nematic phase.

Owing to its own LC nature, this azo compound can be solubilized in LCs in large amount without phase separation. As an example, Figure S2 shows the heating curves of the nematic LC host used in this study, BL006, and its mixture with $15 \%$ of the azo dopant. It can be seen that the miscibility with the LC azo dopant increases the clearing temperature (nematic-isotropic transition temperature, $\mathrm{T}_{\mathrm{ni}}$ ) of BL006 by about $16{ }^{\circ} \mathrm{C}$. The occurrence of the reversible trans-cis photoisomerization of the azo dopant dissolved in BL006 could be confirmed through UV-vis measurements. Figure S3 shows a series of

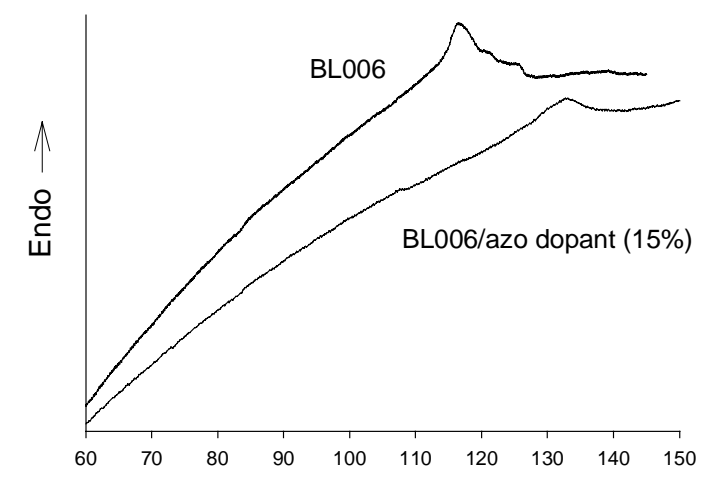

Figure S2. DSC heating curves of BL006 and its mixture with $15 \%$ of azo dopant.
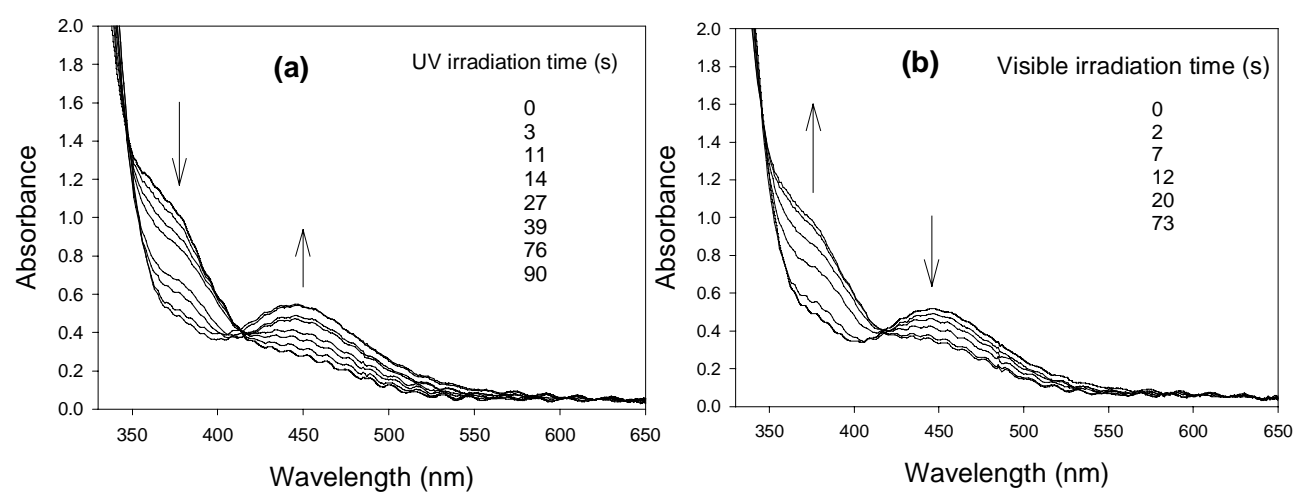

Figure S3. UV-vis spectra of the BL006/dopant (15\%) mixture filled in an ITO-coated cell of $5 \mu \mathrm{m}$ gap: (a) upon UV light irradiation $\left(6.5 \mathrm{~mW} \mathrm{~cm}^{-2}\right)$, and (b) upon visible light irradiation $(4.5 \mathrm{~mW}$ $\left.\mathrm{cm}^{-2}\right)$.

UV-vis spectra of the BL006/azo dopant (15\%) mixture filled in an ITO-coated cell (5 $\mu \mathrm{m})$. Upon UV irradiation $\left(365 \mathrm{~nm}, 6.5 \mathrm{~mW} \mathrm{~cm}^{-2}\right.$ ), the absorption band of trans-azobenzene around $365 \mathrm{~nm}$ decreases, while the absorption band of cis-azobenzene around $450 \mathrm{~nm}$ increases continually with irradiation time, indicating the increase in the amount of cis-azobenzene as a result of the trans-cis isomerization. The opposite change can be noticed on visible light irradiation $\left(440 \mathrm{~nm}, 4.5 \mathrm{~mW} \mathrm{~cm}{ }^{-2}\right)$ due to the reverse cis-trans isomerization. The amount of cis-azobenzene could be calculated from $\mathrm{Cis} \%=\left(1-\mathrm{A} / \mathrm{A}_{o}\right) \times 100$, where $A_{o}$ and $A$ are the absorbance of trans-azobenzene at $365 \mathrm{~nm}$ before and upon irradiation respectively. Irradiation of the mixture was performed using an UV and visible spot curing system (Novacure 2100) combined with interference filters (10 nm bandwidth, Oriel) for UV light $(\lambda=365 \mathrm{~nm})$ and visible light $(\lambda=440 \mathrm{~nm})$. 


\section{Photochemical phase separation observed on polarizing optical microscope}

As the photochemical phase separation occurs, LC molecules are separated from cis-azo dopant and can be realigned by rubbed surfaces (state 3 ). This ordered state could be observed on polarizing optical microscope (POM). Figure S4 shows the photomicrographs of the mixture with $15 \%$ of azo dopant at state 3 recorded with the rubbing direction set at $45^{\circ}$ and $0^{\circ}$ to one of the crossed polarizers. The realignment of LC molecules along the rubbing direction is revealed by the extinction of light transmission at $0^{\circ}$. Inside the cell, no LC textures could be observed due to the alignment. On nonrubbed surfaces, nematic textures could be observed, as shown in Figure S5 for the initial mixture (15\% azo dopant) cast on a non-treated microscope glass slide, and after the photochemical phase separation (state 3). All photomicrographs were taken at room temperature.

(a)

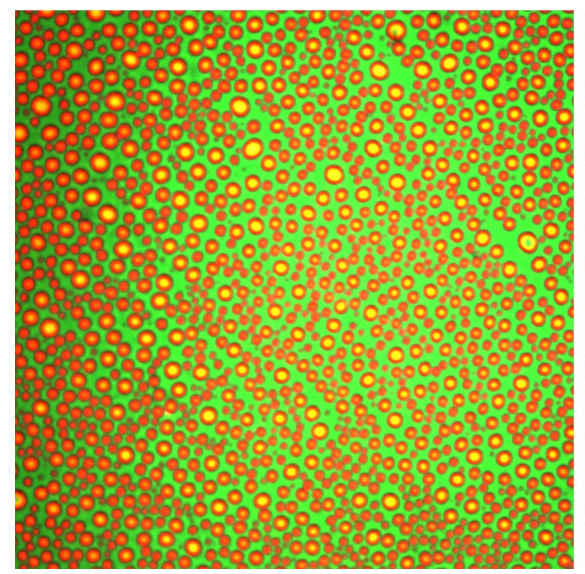

(b)

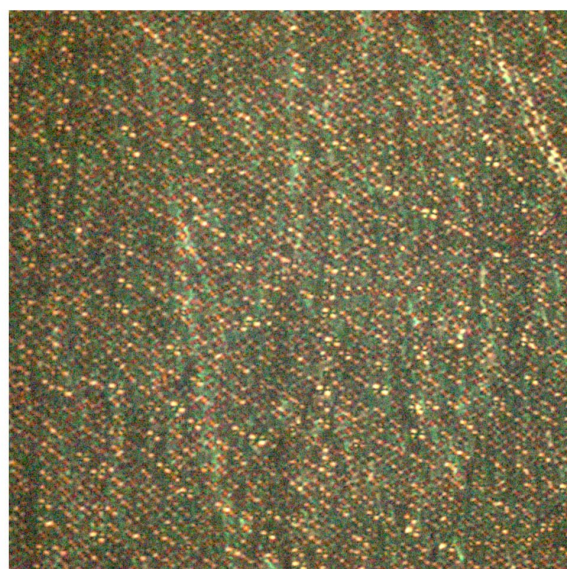

Figure S4. Polarizing optical micrographs of the photochemically phase separated mixture of BL006/dopant (15\%) in a cell with the rubbing direction set at (a) $45^{\circ}$ and (b) $0^{\circ}$ to one of the crossed polarizers. Image area: $156 \mu \mathrm{m} \times 156 \mu \mathrm{m}$

(a)

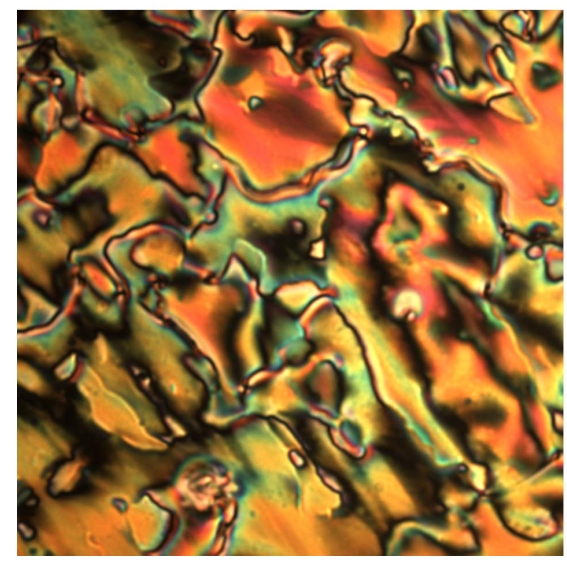

(b)

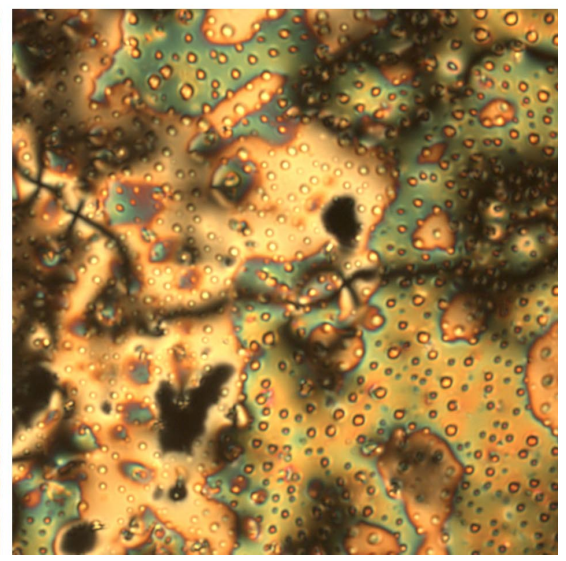

Figure S5. Polarizing optical micrographs of (a) the initial mixture of BL006/dopant (15\%) cast on a glass slide, and (b) after the photochemical phase separation. Image area: $156 \mu \mathrm{m} \times 156 \mu \mathrm{m}$ 


\section{Photochemical effects in the regime of low concentrations of azo dopant}

Figure S6 shows the change in transmittance (through crossed polarizers) of BL006 doped with 5\% of the azo dopant under the same irradiation conditions as for BL006 doped with 15\% of azo dopant (Fig.1b). When UV light was applied, the transmittance dropped as a result of the photochemical phase transition. This disordered low-transmittance state (state 2) was stable under continuous UV irradiation; no photochemical phase separation occurred. When the irradiation was shifted to visible light, the hightransmittance state (state 1) was recovered. This is the typical behavior known for azo dye-doped LCs in the regime of low concentrations of azo dopant.

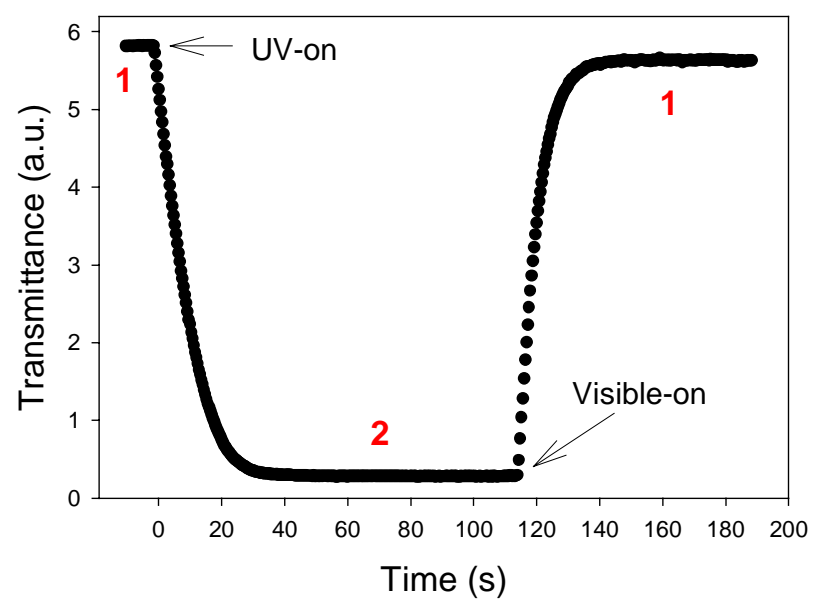

Figure S6. Change in transmittance of BL006/dopant (5\%) exposed to UV ((365 $\left.\mathrm{nm}, 6.5 \mathrm{~mW} \mathrm{~cm}^{-2}\right)$ and visible light ((440 $\left.\mathrm{nm}, 4.5 \mathrm{~mW} \mathrm{~cm}^{-2}\right)$. States 1 and 2 are defined in the paper.

\section{Photocontrolled switching of transmittance in the regime of high concentrations of azo dopant} Both normal and reverse mode of reversible photocontrolled switching of transmittance was demonstrated using the mixture of BL006/azo dopant (15\%) (Fig.2). The transmittance fall-time and rise-time, defined as the time required for change between 10 and $90 \%$ of the initial and of the final level, respectively, were measured under various UV and visible irradiation intensities for the two modes, the results are reported in Figure S7. For both modes, the switching times decrease with increasing the irradiation intensity. The rise-time (to high-transmittance state) is longer than the falltime (to low-transmittance); the difference, however, is much larger for the reverse mode. It is also seen that the fall-time of the reverse mode is shorter that that of the normal-mode, but the opposite can be noticed for the rise-time. For the switching between states 2 and 3, in principle, the phase separation and rehomogenization are processes with similar diffusion-controlled kinetics. One possible explanation for the different switching times is that the measured transmittance fall-time and rise-time should be determined by the actual speeds with which the disordering and ordering of LC molecules take place inside the cell. Theses speeds may be different because the disordering is caused by the perturbation effect exerted by cis-azo molecules while the LC ordering is induced by the effect of rubbed surfaces. More studies are needed to fully understand the differences in the switching times. 


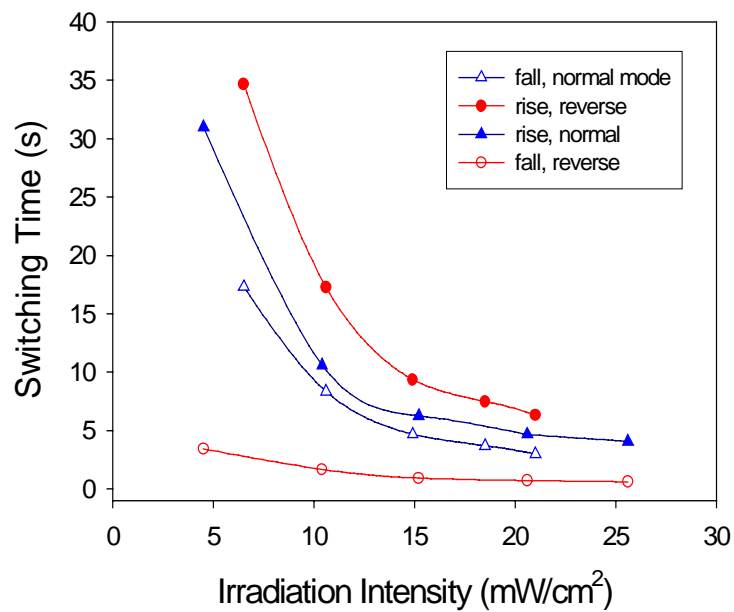

Figure S7. Fall-time and rise-time vs. irradiation intensity for the two modes of photocontrolled switching of transmittance using the mixture of BL006/dopant (15\%).

\section{References}

1. Mariam, Y.; Pemawansa, K.; Okoh, F.; Kofi, B. Mol. Cryst. Liq. Cryst. 1986, 141, 77.

2. Kunitake, T.; Okahata, Y.; Shimomura, M.; Yasunami, S.; Takarabe, K.; J. Am. Chem. Soc. 1981, $103,5401$. 